\title{
Termorregulación, termotolerancia y tasa metabólica de adultos de Macrobrachium tenellum
}

\section{Thermoregulation, thermotolerance and metabolic rate of Macrobrachium tenellum adults}

\author{
Pedro Hernández-Sandoval ${ }^{1,3}$, Viridiana Peraza Gómez ${ }^{2 *}$, Isaac Bacasegua-Villegas ${ }^{3}$, Eliazar \\ Armenta-Valenzuela ${ }^{3}$, Carmen Martínez-Valenzuela ${ }^{3,4}$, Jesús Alanis-Escalante ${ }^{3}$, José López-Sánchez ${ }^{1}$, \\ Marcelo García-Guerrero 5 \\ ${ }^{1}$ Programa de Posgrado en Ciencias Biológico Agropecuarias (CBAP), Universidad Autónoma de Nayarit, Cd. de La Cultura \\ Amado Nervo s/n, CP. 63255, Tepic, Nayarit, México \\ ${ }^{2}$ Escuela Nacional de Ingeniería Pesquera, Universidad Autónoma de Nayarit, Bahía Matanchén Km 12, Carretera a los Cocos, \\ CP. 63740, San Blas, Nayarit, México \\ ${ }^{3}$ Unidad Los Mochis de la Universidad de Occidente, Departamento de Ciencias Biológicas, Boulevard Macario Gaxiola s/n, CP. \\ 81217, Los Mochis, Sinaloa, México. \\ ${ }^{4}$ Instituto de Investigación en Ambiente y Salud, Universidad de Occidente, Gabriel Leyva 169 Sur, Los Mochis, Sinaloa, \\ México. \\ ${ }^{5}$ Laboratorio de Acuacultura, CIIDIR-IPN Oaxaca, Calle Hornos 1003, Col. Noche Buena, CP. 71230, Santa Cruz Xoxocotlán, \\ Oaxaca, México. \\ *Autor de correspondencia: viridianaperaza Qhotmail.com
}

Nota científica recibida: 09 de abril de 2017 aceptada: 31 de octubre de 2017

RESUMEN. El objetivo fue determinar la termorregulación, termotolerancia $\left(\mathrm{TC}_{\max }\right.$ y $\left.\mathrm{TC}_{\min }\right)$ y consumo de oxígeno de adultos de Macrobrachium tenellum $(21.3 \mathrm{~g} \pm 1.23 \mathrm{~g})$, para lo cual se evaluaron cinco temperaturas de aclimatación durante $30 \mathrm{~d}$. La preferencia térmica fue de $26.5^{\circ} \mathrm{C}$, la temperatura de aclimatación no tuvo efecto significativo en las preferencias térmicas. La $\mathrm{TC}_{\min }$ y $\mathrm{TC}_{\max }$ para cada temperatura de aclimatación fue de $10.2{ }^{\circ} \mathrm{C}, 11.01{ }^{\circ} \mathrm{C}, 11.9$ ${ }^{\circ} \mathrm{C}, 12.9{ }^{\circ} \mathrm{C}$ y $13.7{ }^{\circ} \mathrm{C}$, y $36.3{ }^{\circ} \mathrm{C}, 37.5{ }^{\circ} \mathrm{C}, 39.25{ }^{\circ} \mathrm{C}, 40.2{ }^{\circ} \mathrm{C}$ y $41.5^{\circ} \mathrm{C}$, con diferencias significativas entre ellas, mientras que el polígono térmico fue de $331{ }^{\circ} \mathrm{C}^{2}$, característica de especies euritérmicas. La tasa de consumo de oxígeno incrementó de 91.4 a $130.7 \mathrm{mg} \mathrm{O}_{2} \mathrm{~kg}^{-1} \mathrm{~h}^{-1}$ con la temperatura de aclimatación, lo que explica la preferencia de temperaturas bajas, su patrón de distribución y su óptimo térmico en etapa adulta.

Palabras clave: Polígono térmico, acuicultura, temperatura critica, preferendum final

ABSTRACT. The objective was to determine the thermoregulation, thermotolerance $\left(\mathrm{TC}_{\max }\right.$ and $\left.\mathrm{TC}_{\min }\right)$ and oxygen consumption of Macrobrachium tenellum adults $(21.3 \mathrm{~g} \pm 1.23 \mathrm{~g})$, for which five acclimation temperatures were assessed for $30 \mathrm{~d}$. The thermal preference was $26.5{ }^{\circ} \mathrm{C}$; the acclimation temperature had no significant effect on thermal preferences. The $\mathrm{TC}_{\min }$ and $\mathrm{TC}_{\max }$ for each acclimation temperature was $10.2{ }^{\circ} \mathrm{C}, 11.01{ }^{\circ} \mathrm{C}, 11.9{ }^{\circ} \mathrm{C}, 12.9$ ${ }^{\circ} \mathrm{C}$ and $13.7{ }^{\circ} \mathrm{C}$, and $36.3{ }^{\circ} \mathrm{C}, 37.5{ }^{\circ} \mathrm{C}, 39.25{ }^{\circ} \mathrm{C}, 40.2{ }^{\circ} \mathrm{C}$ and $41.5^{\circ} \mathrm{C}$, with significant differences between them, while the thermal polygon was $331^{\circ} \mathrm{C}^{2}$, characteristic of eurythermic species. The oxygen consumption rate increased from 91.4 to $130.7 \mathrm{mg} \mathrm{O}_{2} \mathrm{~kg}^{-1} \mathrm{~h}^{-1}$ with acclimation temperature, which explains the preference for low temperatures, its distribution pattern and its thermal optimum in adult stage.

Key words: Thermal polygon, aquaculture, critical temperature, preferendum final

\section{INTRODUCCIÓN}

Macrobrachium tenellum (Smith 1971) es un langostino que desde hace décadas ha sido objeto de pesca artesanal y de subsistencia en poblaciones cercanas a ríos y zonas costeras de México, El Sal- 


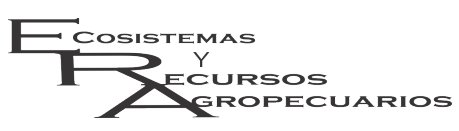

Hernández-Sandoval et al.

Termorregulación en Macrobrachium tenellum

Ecosist. Recur. Agropec.

5(14):353-363,2018

vador, Guatemala y otros países donde se desarrolla (Cabrera 1983). En la naturaleza se encuentra en altas densidades, no es agresivo y puede tolerar las variaciones ambientales (Ponce-Palafox et al. 2013). Siendo una de esas variables la temperatura, factor que afecta a los sistemas biológicos en distintos niveles de organización (Johnston y Bennett 1996), factor que dentro de ciertos valores favorece la producción, pero en valores por arriba del optimo térmico, afecta la salud de los organismos, la demanda de oxígeno y favorece la proliferación, invasión y virulencia de patógenos (Wedemeyer et al. 1999). Desde otra perspectiva, la temperatura juega un papel importante en el establecimiento de los límites de distribución y la supervivencia (Chown 2001). El intervalo de temperaturas preferenciales es una respuesta específica de cada especie, la cual puede variar con la etapa del ciclo de vida, la disponibilidad de alimento, la estacionalidad, la condición patológica, la competencia ecológica, la calidad del agua y la intensidad luminica (Healy y Schulte 2003). La vulnerabilidad a la temperatura depende de los límites térmicos de los organismos y de su capacidad de aclimatación, la cual se desconoce para la mayoría de las especies (Vinagre et al. 2016). La descripción de las condiciones óptimas de los organismos, es indispensables para su mantenimiento en cautiverio, sobre todo si los requerimientos cambian con la edad (Lagerspetz y Vainio 2006).

La tolerancia térmica se puede determinar estableciendo los límites térmicos críticos máximos $\left(\mathrm{TC}_{\max }\right)$ y mínimos $\left(\mathrm{TC}_{\min }\right)$, aumentando o disminuyendo la temperatura de forma gradual hasta llegar a la temperatura en donde se expresen fallas fisiológicas, como la pérdida del equilibrio y espasmos musculares (Terblanche et al. 2007, Díaz et al. 2007). Este método requiere pocos animales, es rápido y sus resultados son comparables en condiciones naturales (Mora y Maya 2006). En este tipo de bioensayos, también se observa la aparición de metabolismo anaerobio, el cual es consecuencia del desequilibrio en la demanda de oxígeno y la capacidad para tomarlo (Frederich y Pörtner 2000). La termotolerancia pude representarse por medio de un polígono térmico, que es una representación gráfica que delimita el nicho térmico de los organismos (Eme y Bennett 2009). Provee ideas importantes de la ecología y la distribución de los organismos y sirve para identificar las tácticas de supervivencia relacionadas con la temperatura, cuantifica el nicho térmico de especies en peligro y ayuda a determinar los óptimos térmicos para su cultivo (Reyes et al. 2011, Madeira et al. 2012).

El consumo de oxígeno es una respuesta fisiológica que se puede correlacionar con las variaciones de los factores ambientales, por lo que la tasa respiratoria, medida en términos de consumo de oxígeno es un indicador de la estimación de la tasa metabólica de crustáceos (Valdez et al. 2008). Uno de los criterios más importantes para determinar el potencial acuícola de una especie es que debe tener una amplia tolerancia a los cambios de temperatura. Por lo anterior, el objetivo del presente trabajo fue determinar la termorregulación, la tolerancia térmica y la respuesta metabólica de adultos de Macrobrachium tenellum aclimatados a diferentes temperaturas.

\section{MATERIALES Y MÉTODOS}

Se colectaron con atarrayas y redes cónicas de mano, 200 organismos de Macrobrachium tenellum en canales de riego para agricultura del municipio de Guasave, Sinaloa ( $25^{\circ} 24^{\prime} 12.50^{\prime \prime}$ LN y $108^{\circ} 27^{\prime}$ 28.07" LO). Los cuales se trasladaron al laboratorio de acuicultura de la Universidad de Occidente, Unidad Los Mochis, para su aclimatación se mantuvieron a temperatura ambiente por $15 \mathrm{~d}$ en una tanque de $5000 \mathrm{~L}$. Todos los días se les proporcionó alimento para camarón (Rangen, 35\% PC) y filete de tilapia fresca (Oreochromis aureus) ad libitum a las 10 y $16 \mathrm{~h}$; las heces, exhuvias y el alimento no consumido se removieron por las mañanas mediante sifoneo, se recuperó los niveles de agua y se proporcionó aireación de forma constante. Cada tercer día se realizó el recambio del $25 \%$ del volumen total de agua, después de la pre-aclimatación se efectuó una biometría preliminar para seleccionar 60 organismos con peso promedio de $21.3 \pm 1.23 \mathrm{~g}$. 


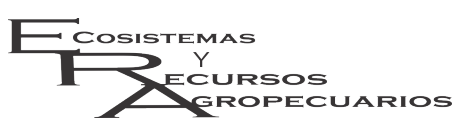

Se utilizó un diseño completamente al azar, colocando tres especímenes por acuario de $60 \mathrm{~L}$, a los que se aplicaron las temperaturas de 20, 23, 26, 29 y $32{ }^{\circ} \mathrm{C}$, las cuales se encuentran dentro del intervalo natural de variación anual en la región. La aireación se proporcionó de forma constante $(>5$ $\left.\mathrm{mgO}_{2} \mathrm{~L}^{-1}\right)$, y se midió con un oxímetro YSI 55. Como alimento se administró filete de tilapia ( $O$. aureus) todos los días a las 10 y $16 \mathrm{~h}$, proporcionando el $10 \%$ de su biomasa. Todos los días se recuperaron los niveles de agua y cada tercer día se realizó el recambio del $25 \%$ del volumen total de agua. Para mantener la temperatura estable se utilizaron calentadores automáticos de $300 \mathrm{~W}$ (Biopro $\pm 1{ }^{\circ} \mathrm{C}$ ), con excepción de la temperatura de $20{ }^{\circ} \mathrm{C}$, la cual se mantuvo con clima ambiental artificial, la temperatura en cada acuario se midió con un termómetro de mercurio convencional, cada tratamiento se realizó por duplicado, las condiciones de aclimatación se mantuvieron durante $30 \mathrm{~d}$ (Manush et al. 2004).

El preferendum térmico de los langostinos se calculó por el método agudo, descrito por Reynolds y Casterlin (1979), se utilizó un gradiente térmico horizontal como el descrito por Díaz et al. (2006), que consta de un tubo de PVC hidráulico de 300 $\mathrm{cm}$ de longitud y $20 \mathrm{~cm}$ de diámetro con $20 \mathrm{seg}$ mentos de $15 \mathrm{~cm}$ de longitud cada uno. La profundidad de la columna de agua fue de $12 \mathrm{~cm}$ y el gradiente se formó al colocar dos calentadores de $300 \mathrm{~W}$ en uno de los extremos del gradiente, mientras que en el extremo opuesto se introdujo hielo en recipientes herméticos. El gradiente tuvo un intervalo de temperatura de 11 a $37^{\circ} \mathrm{C}\left( \pm 1.5^{\circ} \mathrm{C}\right)$. En cada segmento se colocó una piedra aireadora para mantener una concentración de oxígeno disuelto de 5 a $9 \mathrm{mgO}_{2} \mathrm{~L}^{-1}\left(\mathrm{YSI}-55 \pm 0.1{ }^{\circ} \mathrm{C}\right.$ ) y evitar la estratificación térmica de la columna de agua, la temperatura se midió en cada segmento con termómetros de mercurio $\left(-20\right.$ a $\left.100^{\circ} \mathrm{C}\right)$. Se utilizaron tres organismos en cada condición experimental, los cuales fueron colocados de forma individual en el segmento con la temperatura similar a la de aclimatación. La ubicación de cada organismo y temperatura de cada cámara se registraron cada $10 \mathrm{~min}$
Hernández-Sandoval et al. Termorregulación en Macrobrachium tenellum Ecosist. Recur. Agropec. 5(14):353-363,2018

durante $2 \mathrm{~h}$, cada organismo se consideró como una réplica $(N=15)$. El preferendum final se determinó gráficamente (Reynolds y Casterling 1979), por medio de la intersección de las temperaturas preferidas por los organismos provenientes de cada temperatura de aclimatación ( $y=a+b x)$.

Para el cálculo de las temperaturas criticas máximas $\left(\mathrm{TC}_{\max }\right)$, se utilizaron tres organismos $(\mathrm{N}$ $=15)$ provenientes de cada temperatura de aclimatación $\left(20,23,26,29\right.$ y $\left.32{ }^{\circ} \mathrm{C}\right)$, los cuales se colocaron de uno a la vez en acuarios de $24 \mathrm{x}$ $12 \times 12 "$ (45 L), que tenian una resistencia eléctrica de $900 \mathrm{~W}$ (Steren ${ }^{\circledR}$, WL-1300) y una bomba sumergible (MagiDeal Ac 110v-5w) para recircular el agua y mantener la temperatura homogénea, los langostinos se colocaron 30 min antes de dar inicio al experimento para disminuir el estrés por manejo (Díaz et al. 2002, Pérez et al. 2003). Para luego incrementar la temperatura del agua a una tasa de $1{ }^{\circ} \mathrm{C} \mathrm{m}^{-1}$ hasta observar la perdida de equilibrio (PE) (Re et al. 2005), e inmediatamente tomar nota de la temperatura a la cual se observó la respuesta. Los langostinos se depositaron de nuevo en la temperatura de aclimatación. De la misma forma, para la temperatura critica mínima $\left(\mathrm{TC}_{\min }\right)$ se colocaron los langostinos en un cilindro de malla rígida de $15 \mathrm{~cm}$ de largo y $10 \mathrm{~cm}$ de diámetro, en el gradiente térmico horizontal en el segmento similar a la temperatura de aclimatación y se desminuyo $1{ }^{\circ} \mathrm{C} \mathrm{m}^{-1}$, hasta observar la $\mathrm{PE}$, tomando nota de la temperatura, para luego devolver al langostino a la temperatura de aclimatación. El polígono de tolerancia térmica se elaboró a partir de la unión de las medias de los límites críticos térmicos máximos y mínimos $\left(\mathrm{TC}_{\max }\right.$ y $\left.\mathrm{TC}_{\min }\right)$. La tasa de respuesta de aclimatación (TRA) se obtuvo con los datos obtenidos de $\mathrm{TC}_{\max }$ y $\mathrm{TC}_{\text {min }}$ divididos entre la temperatura de aclimatación (Claussen 1977).

Se tomaron tres organismos de cada condición experimental $(\mathrm{N}=15)$, y se colocaron de forma individual en matraces kitazato de $1 \mathrm{~L}$ que funcionaron como cámaras respirométricas, en un sistema respirométrico semiabierto (Figura 1). Una cámara vacía se utilizó como control para corregir el consumo de oxígeno ocasionado por 


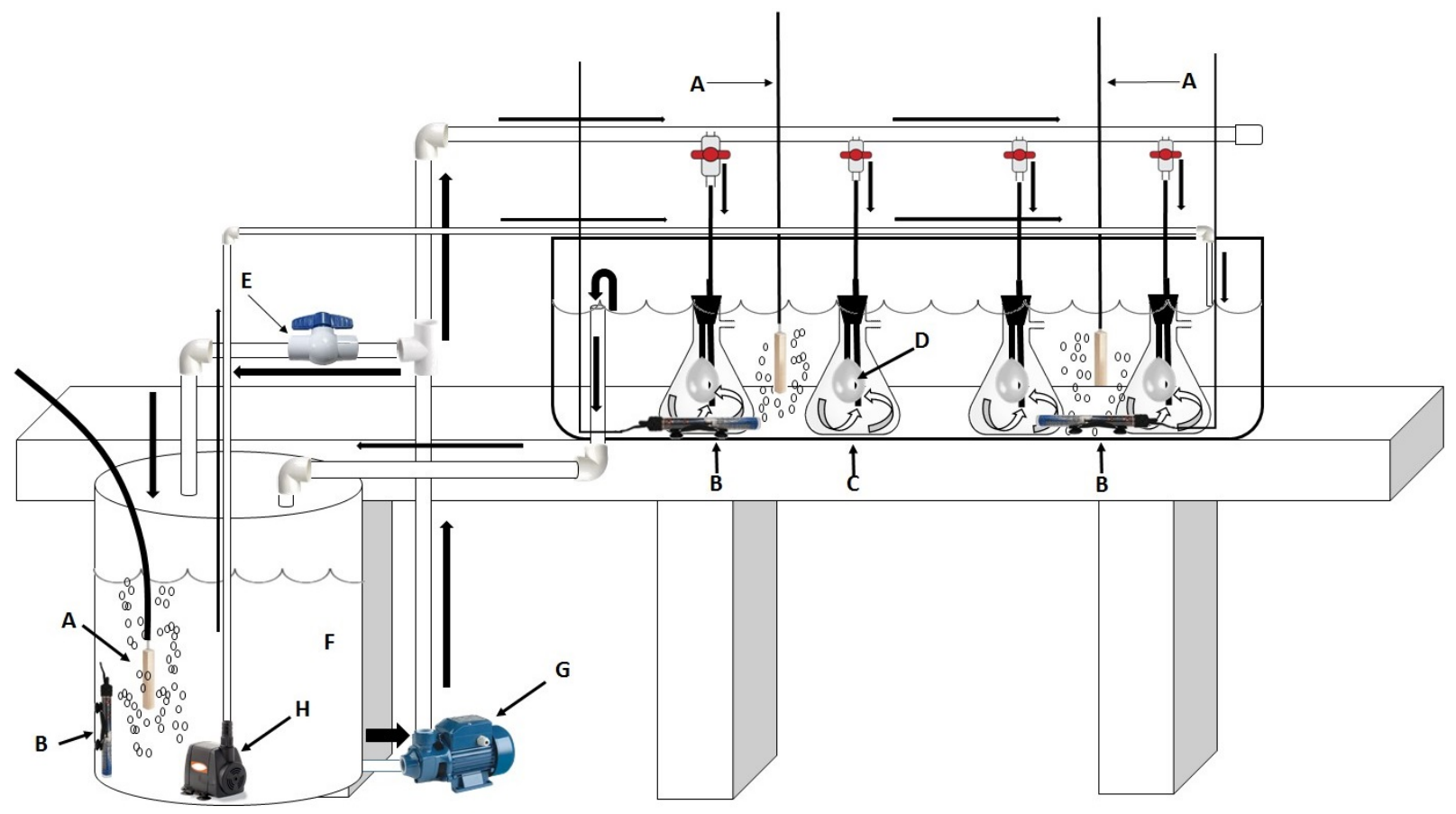

Figura 1. Sistema respirométrico semiabierto. A) Piedras aireadoras, B) Calentadores automáticos (Biopro $\pm 0.5^{\circ} \mathrm{C}, 300 \mathrm{~W}$ ), C) Cámaras respirométricas $1 \mathrm{~L}, \mathrm{D})$ Globo, E) Válvula de desahogo, F) Reservorio de agua, G) Bomba periférica $\left(0.5\right.$ HP-Truper $\left.{ }^{\circledR}\right)$ y $\mathrm{H})$ Bomba sumergible (Evans $30 \mathrm{~W}^{\circledR}$ ).

microorganismos presentes en el sistema. Los langostinos no se alimentaron $24 \mathrm{~h}$ antes de iniciar la determinación. El flujo del agua permaneció abierto por $2 \mathrm{~h}$ en la cámara respirométrica (Pérez et al. 2003), para determinar la concentración de oxígeno inicial, luego se cerró el flujo por $1 \mathrm{~h}$ para evitar que la concentración disminuyera al 30\%, y se convirtiera en un factor de estrés (Stern et al. 1984). Para luego tomar una muestra de agua a la que se le determinó la concentración final de oxígeno disuelto. Las mediciones se realizaron a intervalos de una hora entre las 9 y $13 \mathrm{~h}$, realizando las mediciones por duplicado.

La concentración inicial y final de oxígeno disuelto se realizó con un oxímetro YSI 52 (Yellow Spring Instruments) provisto de un sensor polarográfico con precisión de $\pm 0.03 \mathrm{~mL}^{-1}$. El consumo de oxígeno se calculó como la diferencia entre la concentración inicial y final de cada cámara en cada temperatura de aclimatación, expresándose como $\mathrm{mg} \mathrm{O}_{2} \mathrm{~kg}^{-1} \mathrm{~h}^{-1}$, con la formula siguiente (Cerezo et al. 2006, Zheng et al. 2008): $T C O=$ $\left(C_{t}-C_{0}\right) V /(W x T)$. Donde: $C_{t}$ es el cambio en la concentración de oxígeno en la cámara antes y después de la prueba, $C_{0}$ es la diferencia en oxígeno en la cámara blanco (control), $V$ es el volumen de la cámara, $W$ es el peso del langostino en $\mathrm{kg}, T$ es el tiempo en horas, y TCO es la tasa de consumo de oxígeno.

Se aplicaron pruebas de normalidad (ShapiroWilk, $p>0.05)$ y homogeneidad de varianza $(p>$ 0.05 ) a los de datos, mientras que a las variables no paramétricos se les aplicó la prueba KruskalWallis $(p<0.05)$, a los resultados con diferencias estadísticas significativas se realizo un análisis de comparaciones múltiples post hoc de Student Newman Keuls ( $p<0.05$, van-Emden 2008), los datos se graficaron en cajas en paralelo, los análisis se realizarón con el software Sigma plot v.12.5. 
Tabla 1.Temperatura Crítica Mínima, Máxima y temperatura preferida en adultos de Macrobrachium tenellum aclimatados a diferentes temperatura (letras distintas indican diferencias estadísticas significativas; Media \pm E.S.; Student Newman Keuls, $\mathrm{p}<0.05)$.

\begin{tabular}{cccc}
\hline $\begin{array}{c}\text { Temperatura de } \\
\text { aclimatación } \\
\left(\mathrm{T}_{\mathrm{A} .}{ }^{\circ} \mathrm{C}\right)\end{array}$ & $\begin{array}{c}\text { Temperatura Critica } \\
\text { Mínima } \\
\left(\mathrm{TC}_{\min }{ }^{\circ} \mathrm{C}\right)\end{array}$ & $\begin{array}{c}\text { Temperatura Crítica } \\
\text { Máxima } \\
\left(\mathrm{TC}_{\max }{ }^{\circ} \mathrm{C}\right)\end{array}$ & $\begin{array}{c}\text { Temperatura } \\
\text { Preferida } \\
\left(\mathrm{T}^{\circ} \mathrm{P} .{ }^{\circ} \mathrm{C}\right)\end{array}$ \\
\hline 20 & $10.4 \pm 0.29^{a}$ & $36.4 \pm 0.099^{a}$ & $26.03 \pm 0.62$ \\
23 & $11.1 \pm 0.19^{b}$ & $37.5 \pm 0.26^{b}$ & $28.05 \pm 0.42$ \\
26 & $12.1 \pm 0.21^{c}$ & $39.2 \pm 0.51^{c}$ & $27.05 \pm 0.43$ \\
29 & $13.3 \pm 0.29^{d}$ & $40.8 \pm 0.24^{d}$ & $26.69 \pm 0.57$ \\
32 & $13.9 \pm 0.51^{e}$ & $41.9 \pm 0.25^{e}$ & $26.86 \pm 0.58$ \\
\hline
\end{tabular}

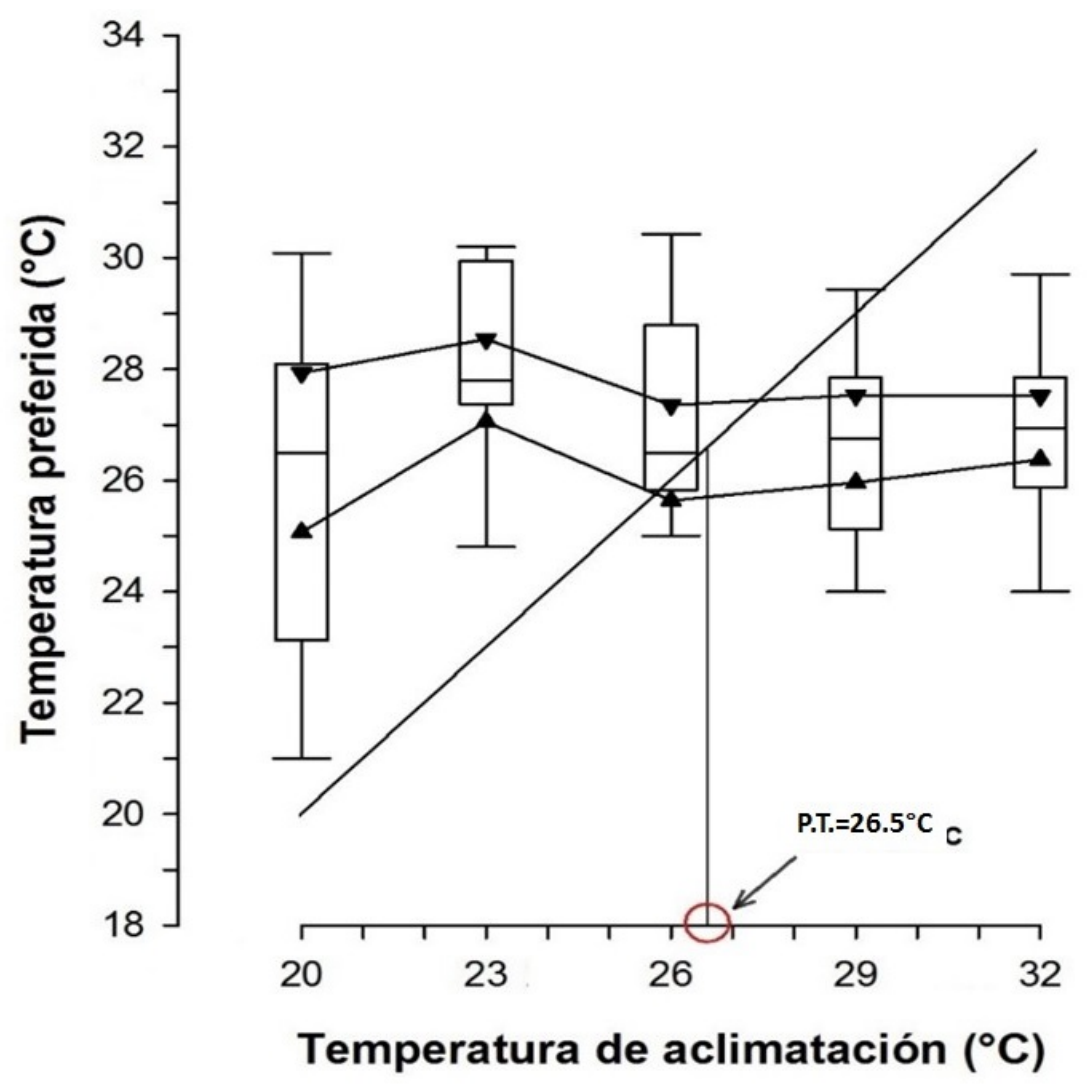

Figura 2. Preferencia térmica en adultos de Macrobrachium tenellum aclimatados a diferentes temperaturas. La zona bordeada por los triángulos representa el $95 \%$ del intervalo de confianza de la mediana. Las líneas y barras verticales representan los cuartiles (Kruskall Wallis, $\mathrm{p}>0.05$ ).

\section{RESULTADOS Y DISCUSIÓN}

Se calculó un intervalo de temperaturas preferidas de 26.03 a $28.05{ }^{\circ} \mathrm{C}$ (Tabla 1), con preferendum térmico final de los adultos de $M$. tenellum de $26.5{ }^{\circ} \mathrm{C}$ (Figura 2), el cual es menor a $28.5{ }^{\circ} \mathrm{C}$ y $32.2{ }^{\circ} \mathrm{C}$ reportados por Hernández et al. (1995) para juveniles y Flores et al. (2012) para postlarvas de la misma especie. Lo que concuerda con la tercera hipótesis de Giattina y Garton (1982) quienes indican que la temperatura preferida puede reflejar el óptimo térmico para que se realicen de forma adecuada los procesos fisiológicos. Al respecto, Wedemeyer et al. (1999) mencionan que 


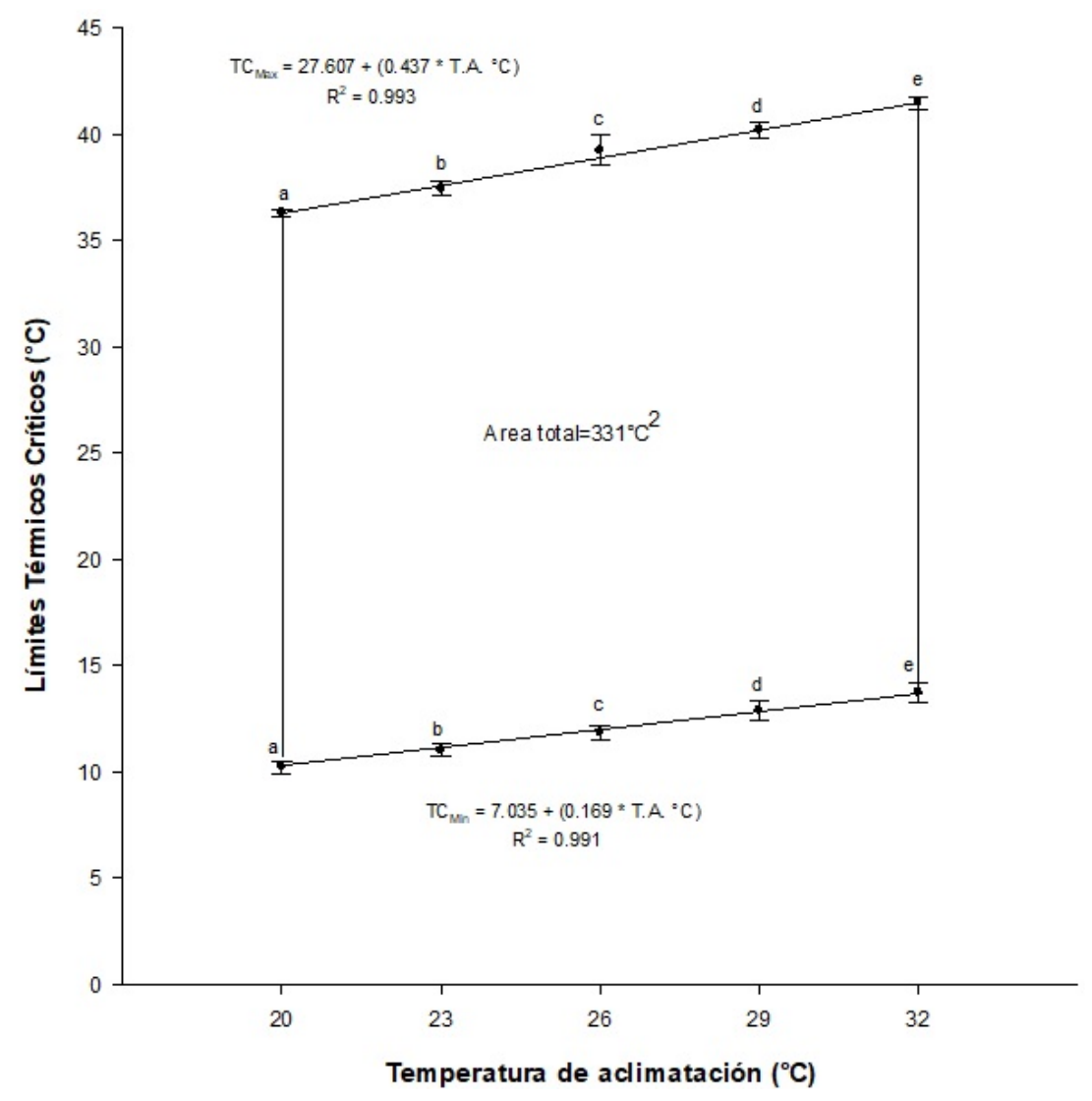

Figura 3. Polígono de termotolerancia de adultos de Macrobrachium tenellum; área total $331{ }^{\circ} \mathrm{C}^{2}$. En $\mathrm{TC}_{\max }$ y $\mathrm{TC}_{\min }$ letras distintas indican diferencias estadísticas significativas (Media \pm E.S.; Student Newman Keuls, $p<0.05$ ), también se muestra el modelo de regresión lineal simple de la $\mathrm{TC}_{\max } \mathrm{y} \mathrm{TC}_{\min }$ en función de la temperatura de aclimatación.

la preferencia térmica es una respuesta especieespecífica que puede variar con el tiempo de aclimatación, la edad, el peso, la alimentación, la calidad del agua, la intensidad luminosa y por factores como la competencia y la presencia de patógenos. De acuerdo con Díaz et al. (2015) la temperatura preferida optimiza los procesos fisiológicos y bioquímicos para su máxima eficiencia. Las diferencias con otros trabajos se deben posiblemente a los requerimientos fisiológicos específicos de la edad. Sobre la preferencia térmica González et al. (2010) encontraron que adultos de Litopenaeus vannamei tienen una preferencia térmica de $26.2^{\circ} \mathrm{C}$, valor que es menor que la encontrada reportada en postlarvas y juveniles de 29 a $30{ }^{\circ} \mathrm{C}$ por Díaz y Bückle (1993). Posiblemente la mayor temperatura afecte la salud de los gametos, ocasionando malformaciones de espermatozoides y menor supervivencia. No se encontró relación entre la aclimatación y las preferencias térmicas, pero el efecto de las temperaturas de aclimatación sobre las preferencias térmicas varía entre ectotermos acuáticos (Reiser et al. 2014). Al respecto, Flores et al. (2012) reportan diferencias significativa entre la temperatura de aclimatación y las preferencias térmicas, de postlarvas y juveniles de M. tenellum.

La temperatura máxima se incrementó con la temperatura de aclimatación de 20 a $32{ }^{\circ} \mathrm{C}$, con diferencias estadísticas entre las temperaturas (SNK, p < 0.05). En la Temperatura mínima se observó un incremento a partir de la temperatura de aclimatación, con diferencias estadísticas $(p<$ 


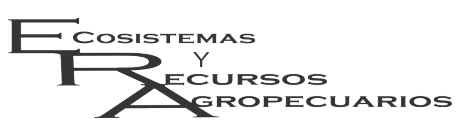

0.05). El polígono de tolerancia térmica alcanzó un área de $331{ }^{\circ} \mathrm{C}^{2}$ (Figura 3). Los valores de TRA para los organismos expuestos a la $\mathrm{TC}_{\max } \mathrm{y}$ $\mathrm{TC}_{\min }$ tuvieron un intervalo de 0.28 a $0.37 \mathrm{y}$ de 0.45 a 0.55 , con promedio de 0.32 y 0.49 , respectivamente (Tabla 2). La amplitud de la zona de tolerancia térmica de una especie puede variar con la etapa de desarrollo, edad o dentro de la misma etapa de desarrollo debido a la plasticidad fenotípica (Bowler y Terblanche 2008). Los polígonos de termotolerancia ayudan en la identificación de estos intervalos, su utilidad radica en que se puede usar como índice, para comparar la termotolerancia para la misma especie en diferentes lugares, o en distintas etapas de su ciclo de vida, lo que resulta en amplitud del rango de distribución, valores óptimos para su cultivo (Portner y Knust 2007, Portner y Farrell 2008). Se encontró un valor de $331^{\circ} \mathrm{C}^{2}$, al respecto mientras Manush et al. (2004) en adultos y juveniles de $\mathrm{M}$. rosenbergii reportaron áreas de $334{ }^{\circ} \mathrm{C}^{2}$ y $318{ }^{\circ} \mathrm{C}^{2}$, respectivamente, mientras que Díaz et al. (2002) reportan $286{ }^{\circ} \mathrm{C}^{2}$ para M. acanthurus. Mientras que Kumlu et al. (2010) obtuvieron para postlarvas de Litopenaeus vannamei un polígono de termotolerancia de $435{ }^{\circ} \mathrm{C}^{2}$.

Tabla 2. Tasa de Respuesta a la Aclimatación (TRA) de adultos Macrobrachium tenellum

\begin{tabular}{lccc}
\hline \multicolumn{4}{c}{ Temperatura de aclimatación $\left({ }^{\circ} \mathrm{C}\right)$} \\
\hline TRA & $20-26$ & $23-29$ & $26-32$ \\
TC $_{\text {min }}$ & 0.28 & 0.37 & 0.30 \\
TC $_{\text {max }}$ & 0.47 & 0.55 & 0.45 \\
\hline
\end{tabular}

La TRA se utiliza como índice de la magnitud de aclimatación térmica (Claussen 1977), en adultos de $M$. tenellum se encontraron valores de 0.28 a 0.37, al respecto Díaz et al. (1998) indican que el valor TRA depende del gradiente de temperatura geográfica. La TRA de los crustáceos sugiere que las especies que normalmente habitan en las regiones tropicales y subtropicales, tienen mayores valores de TRA (Hernández et al. 2006, Kir y Kumlu 2008, Noyola et al. 2016). En diversos trabajos se ha encontrado que las especies que viven en regiones frías y templadas que experimentan cam-
Hernández-Sandoval et al. Termorregulación en Macrobrachium tenellum Ecosist. Recur. Agropec. 5(14):353-363,2018

bios graduales de temperatura a largo plazo tienen el tiempo para hacer los ajustes metabólicos sin cambios sustanciales en sus rangos de tolerancia (Díaz et al. 2002). Por el contrario, especies tropicales y subtropicales como los langostinos experimentan mayores fluctuaciones de temperatura durante cortos periodos de tiempo y deben tener amplios intervalos de tolerancia para sobrevivir a los cambios relativamente rápidos de la temperatura del agua. Esta respuesta es típica de ectotermos acuáticos como lo mencionaron Díaz et al. $(2002,2004)$ y $R e$ et al. $(2005,2006)$. El papel de la temperatura en los procesos biológicos responde en los indicadores de bienestar como la locomoción, crecimiento y reproducción (Angilletta et al. 2002), y efecto en la distribución y abundancia de las especies (Schulte 2015).

La tasa de consumo de oxígeno presentó diferencias estadísticas (SNK, $\mathrm{p}<0.05)$ entre las distintas temperaturas de aclimatación, pero no se encontraron diferencia entre las temperaturas de 26 y $29{ }^{\circ} \mathrm{C}$ (Figura 4). Un estudio sobre el consumo de oxígeno puede proporcionar información valiosa de los verdaderos requisitos de los langostinos (Niu et al. 2003). El consumo de oxígeno y su difusión por medio de la ventilación y la circulación es una habilidad cuya eficiencia depende de la especie, la identificación de las necesidades dentro de los intervalos de termotolerancia y óptimos, proporcionaran los valores ideales para su desarrollo (Pörtner 2002). Dentro de la gama de temperaturas en que esto ocurre, la respiración y la asimilación de nutrientes son las ideales para el crecimiento y supervivencia (Giomi y Portner 2013). El aumento de consumo de oxígeno en función de la temperatura indica la necesidad de oxígeno (Schulte 2015). No se observó disminución en el consumo de oxígeno en función de la temperatura, lo que se pudo deber a que las $\mathrm{TC}_{\max }$ son mayores que la mayor de aclimatación. La tasa de consumo de oxígeno de $123.5688 \mathrm{mgO}_{2} \mathrm{~kg}^{-1} \mathrm{~h}^{-1}$ en la temperatura cercana a la preferida $\left(26.5^{\circ} \mathrm{C}\right)$ es importante para proporcionar la concentración adecuada de oxígeno de los langostinos. Las temperaturas de aclimatación no tuvieron efecto sobre las preferencias térmicas. 


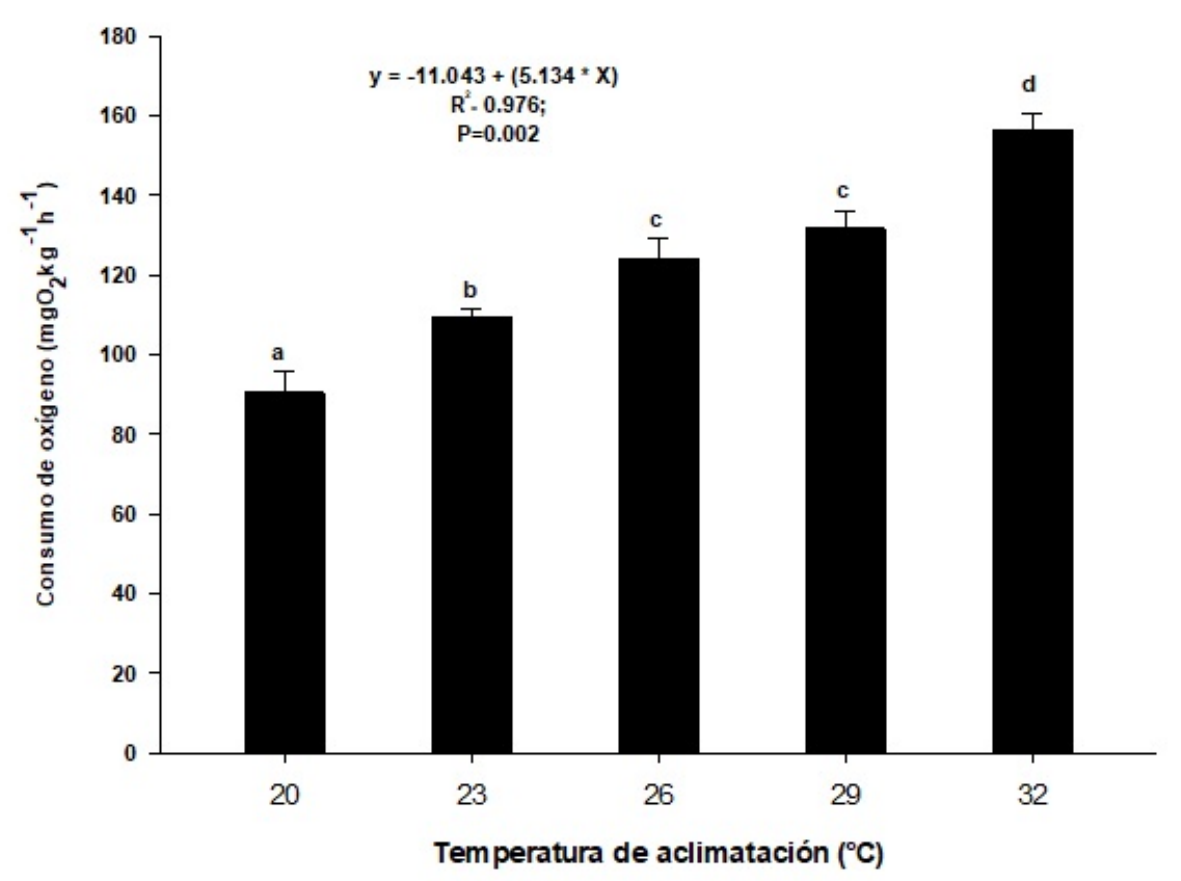

Figura 4. Tasa de consumo de oxígeno $\left(\mathrm{mgO}_{2} \mathrm{~kg}^{-1} \mathbf{h}^{-1}\right)$ de adultos de Macrobrachium tenellum aclimatados a cinco temperaturas. Letras distintas indican diferencias estadísticas significativas (Media \pm E.S.; Student Newman Keuls, p < 0.05).

La temperatura preferida de los adultos de $M$. tenellum fue de $26.5{ }^{\circ} \mathrm{C}$ y el área del polígono de termotolerancia fue de $331{ }^{\circ} \mathrm{C}^{2}$, lo que indica que es una especie euriterma.

\section{LITERATURA CITADA}

Angilletta MJ, Niewiarowski PH, Navas CA (2002) The evolution of thermal physiology in ectotherms. Journal of Thermal Biology 27: 249-268.

Bowler K, Terblanche JS (2008) Insect thermal tolerance: what is the role of ontogeny, ageing and senescence? Biological Reviews 83: 339-55.

Cabrera PJ (1983) Carácter práctico para diferenciación de sexos en Macrobrachium tenellum (Crustacea: Decapoda: Natatia). Revista de Biología Tropical 31: 159-160.

Cerezo VJ, Martínez LFJ, García GB (2006) Oxygen consumption and ventilatory frequency responses to gradual hypoxia in common dentex (Dentex dentex): Basis for suitable oxygen level estimations. Aquaculture 256: 542-551.

Chown SL (2001) Physiological variation in insects: hierarchical levels and implications. Journal of Insect Physiology 47: 649-660.

Claussen DL (1977) Thermal acclimation in ambystomatid salamanders. Comparative Biochemistry and Physiology Part A: Physiology 58A: 333-340.

Díaz F, Bückle LF (1993) Thermoregulatory behaviour of Macrobrachium rosenbergii (Crustacea, Palaemonidae). Tropical Ecology 43: 199-203. 
Díaz F, Sierra E, Buckle LF, Garrido A (1998) Critical thermal maxima and minima of Macrobrachium rosenbergii (Decapoda:Palemonidae). Journal of Thermal Biology 23: 381-385.

Díaz F, Sierra E, Re AD, Rodríguez L (2002) Behavioural thermoregulation and critical thermal limits of Macrobrachium acanthurus (Wiegman). Journal of Thermal Biology 27: 423-428.

Diaz F, Re AD, Sierra E, Amador G (2004) Behavioural thermoregulation and critical limits applied to the culture of red claw Cherax quadricarinatus (von Martens). Freshwater Crayfish 14: 90-98.

Díaz F, Re AD, Medina Z, Valdez G, Valenzuela F (2006) Thermal preference and tolerance of green abalone Haliotis fulgens (Philippi, 1845) and pink abalone Haliotis corrugata (Gray, 1828). Aquaculture Research 37: 877-884.

Díaz F, Re AD, González RA, Sánchez LN, Leyva G, Valenzuela F (2007) Temperature preference and oxygen consumption of the largemouth bass Micropterus salmoides (Lacepéde) acclimated to different temperatures. Aquaculture Research 38: 1387-1394.

Díaz F, Re AD, Salas A, Galindo-Sanchez CE, González M, Sánchez A, et al. (2015) Behavioral thermoregulation and critical thermal limits of giant keyhole limpet Megathura crenulata (Sowerby 1825) (Mollusca; Vetigastropoda). Journal of Thermal Biology 54:133-138.

Eme J, Bennett WA (2009) Critical thermal tolerance polygons of tropical marine fishes from Sulawesi, Indonesia. Journal of Thermal Biology 34: 220-225.

Flores RR, Lazareno MM, Espinoza Ch, Basto RE, Vega VF (2012) Temperatura óptima y preferencia térmica del camarón de río Macrobrachium tenellum en la costa tropical del Pacifico Mexicano. Boletim do Instituto de Pesca 38: 121-130.

Frederich M, Pörtner HO (2000) Oxygen limitation of thermal tolerance defined by cardiac and ventilatory performance in spider crab, Maja squinado. American journal of physiology. Regulatory, integrative and comparative physiology 279: 1531-1538.

Giattina JD, Garton RR (1982) Graphical model of thermoregulatory behavior by fishes with a new measure of eurythermality. Canadian Journal of Fisheries and Aquatic Sciences 39: 524-528.

Giomi F, Pörtner HO (2013) A role for haemolymph oxygen capacity in heat tolerance of eurythermal crabs. Aquatic-physiology 4: 1-13.

González RA, Diaz F, Licea A, Re AD, Sanchez LN, Garcia-Esquivel Z (2010) Thermal preference, tolerance and oxygen consumption of adult white shrimp Litopenaeus vannamei (Boone) exposed to different acclimation temperatures. Journal of Thermal Biology 35: 218-224.

Healy TM, Schulte PM (2003) Thermal Acclimation Is Not Necessary to Maintain a Wide Thermal Breadth of Aerobic Scope in the Common Killifish (Fundulus heteroclitus). Physiological and Biochemical Zoology 85:107-119.

Hernández M, Bückle LF, Diaz F. (1995) Preferred temperature of Macrobrachium tenellum (Crustacea: Palaemonidae). Rivista Italiana di Acquacoltura 30: 93-96.

Hernández M, Buckle LF, Palacios E, Baron B (2006) Preferential behavior of white shrimp Litopenaeus vannamei (Boone 1931) by progressive temperature- salinity simultaneus interaction. Journal of Thermal Biology 31: 565-572.

Johnston IA, Bennett AF (1996) Animals and temperature: Phenotypic and evolutionary adaptation. Cambridge University Press, Cambridge. Englad. 436p. 
Kir M, Kumlu M (2008) Effect of temperature and salinity on low thermal tolerance of Penaeus semisulcatus (Decapoda: Penaeidae). Aquaculture Research 39: 1101-1106.

Kumlu M, Turkmen S, Kumlu M (2010) Thermal tolerance of Litopenaeus vannamei (Crustacea: Penaeidae) acclimated to four temperatures. Journal of Thermal Biology 35: 305-308.

Lagerspetz K, Vainio L (2006) Thermal behavior of crustaceans. Biological Reviews 81: 237-258.

Madeira D, Narciso L, Cabral HN, Vinagre C (2012) Thermal tolerance and potential impacts of climate change on coastal and estuarine organisms. Journal of Sea Research 70:32-41.

Manush SM, Pal AK, Chatterjee N, Das T, Mukherjee SC (2004) Thermal tolerance and oxygen consumption of Macrobrachium rosenbergii acclimated to three temperatures. Journal of Thermal Biology 29: 15-19.

Mora C, Maya M (2006) Effect of the rate of temperature increase of the dynamic method on the heat tolerance of fishes. Journal of Thermal Biology 31: 337-341.

Niu C, Lee D, Goshima S, Nakao S (2003) Effects of temperature on food consumption, growth and oxygen consumption of the freshwater prawn Macrobrachium rosenbergii De Man, 1879. Aquaculture Research 34: 501-506.

Noyola RJ, Mascaró M, Díaz F, Re AD, Sánchez-Zamora A, Caamal-Monsreal C. et al. (2016) Thermal biology of prey (Melongena corona bispinosa, Strombus pugilis, Callinectes similis, Libinia dubia) and predators (Ocyurus chrisurus, Centropomus undecimalis) of Octopus maya from the Yucatan Peninsula. Journal of Thermal Biology 53: 151-161.

Pérez E, Diaz F, Espina S (2003) Thermoregulatory behavior and rritical thermal limits of the angelfish Pterophyllum scalare (Lichtenstein) (Pisces: Cichlidae). Journal of Thermal Biology 28: 531-537.

Ponce-Palafox JT, Uriostegui FL, Benítez-Mandujano MA, Castillo-Vargasmachuca, Benítez-Valles J, GómezGurrola, et al. (2013) Comparative growth performance of male and female fresh- water prawn Macrobrachium tenellum (Decapoda: Palaemonidae) cultured in tropical earthen ponds. International Journal of Fisheries and Aquaculture 5:26-28.

Pörtner HO (2002) Climate variations and the physiological basis of temperature dependent biogeography: systemic to molecular hierarchy of thermal tolerance in animals. Comparative Biochemistry and Physiology A 132:739-761.

Portner HO, Knust R (2007) Climate change affects marine fishes through the oxygen limitation of thermal tolerance. Science 315: 95-97.

Portner HO, Farrell AP (2008) Physiology and climate change. Science 322: 690-692.

Re AD, Díaz F, Sierra E, Rodríguez J, Pérez E (2005) Effect of salinity and temperature on thermal tolerance of Brown shrimp Farfantepenaeus aztecus (Ives) (Crustacea, Penaeidae). Journal of Thermal Biology 30: 618-622.

Re AD, Diaz F, Valdez G (2006) Effect of salinity on the thermoregulatory behavior of juvenile blue shrimp Litopenaeus stylirostris Stimpson. Journal of Thermal Biology 31: 506-513.

Reiser S, Herrmann JP, Temming A (2014) Thermal preference of the common brown shrimp (Crangon crangon L.) determined by the acute and gravitational method. Journal of Experimental Marine Biology and Ecology 461: 250-256.

Reyes I, Díaz F, Re AD, Pérez J (2011) Behavioral thermoregulation, temperature tolerance and oxygen consumtion in the Mexican bullseye puffer fish, Sphoeroides annulatus Jenyns (1842), acclimated to different temperatures. Journal of Thermal Biology 36: 200-205. 
Reynolds WW, Casterlin ME (1979) Behavioral thermoregulation and the final preferendum paradigm American Zoologist 19: 211-224.

Schulte PM (2015) The effects of temperature on aerobic metabolism: towards a mechanistic understanding of the responses of ectotherms to a changing environment. The Journal of Experimental Biology 218: 1856-1866.

Stern S, Borut A, Cohen D (1984) The effect of salinity and ion composition on oxygen consumption and nitrogen excretion of Macrobrachium rosenbergii (De Man). Comparative Biochemistry and Physiology 79: 271-27.

Terblanche JS, Deere JA, Clusella-Trullas S, Janion C, Chown SL (2007) Critical thermal limits depend on methodological context. Proceedings of the Royal Society 274: 2935-2942.

Valdez G, Díaz F, Sierra E (2008) Effect of salinity on physiological energetics of white shrimp Litopenaeus vannamei, Boone. Hidrobiológica 18:105-115.

Van-Emden H (2008) Statistics for terrified biologists. Blackwell Scientific Publications, Cambridge, Massachusetts. 343p.

Vinagre C, Leal I, Mendonca V, Madeira D, Narciso L, Dinis M, et al. (2016) Vulnerability to climate warming and acclimation capacity of tropical and temperate coastal organisms. Ecological Indicators 62: 317-327.

Wedemeyer GR, Meyer FP, Smith L (1999) Environmental stress and fish diseases. Narendra Publishing House. Delhi, India. 107p.

Zheng J, Nakatsuji T, Roer RD, Watson RD (2008) Studies of a receptor guanylyl cyclase cloned from Y-organs of the blue crab (Callinectes sapidus), and its possible functional link to ecdysteroidogenesis. General and Comparative Endocrinology 155: 780-788. 
